\title{
Magnetically controlled exciton transfer in hybrid quantum-dot-quantum-well nanostructures
}

\author{
V. Laurindo Jr., ${ }^{1}$ Yu. I. Mazur, ${ }^{2}$ E. R. Cardozo de Oliveira, ${ }^{1}$ B. Alén, ${ }^{3}$ M. E. Ware, ${ }^{2}$ E. Marega Jr., ${ }^{4}$ Z. Ya. Zhuchenko, \\ G. G. Tarasov, ${ }^{5}$ G. E. Marques, ${ }^{1}$ M. D. Teodoro, ${ }^{1, *}$ and G. J. Salamo ${ }^{2}$ \\ ${ }^{1}$ Departamento de Física, Universidade Federal de São Carlos, 13565-905 São Carlos, São Paulo, Brazil \\ ${ }^{2}$ Institute for Nanoscience and Engineering, University of Arkansas, Fayetteville, Arkansas 72701, USA \\ ${ }^{3}$ Instituto de Micro y Nanotecnología, IMN-CNM, CSIC (CEI UAM + CSIC) Isaac Newton, 8, E-28760, Tres Cantos 28760, Madrid, Spain \\ ${ }^{4}$ Instituto de Física de São Carlos, Universidade de São Paulo, 13.566-590 São Carlos, São Paulo, Brazil \\ ${ }^{5}$ Institute of Semiconductor Physics, National Academy of Sciences, pr, Nauki 45, Kiev 03028, Ukraine
}

(Received 18 February 2019; revised manuscript received 2 July 2019; published 19 July 2019)

\begin{abstract}
A magnetophotoluminescence study of the carrier transfer with hybrid InAs/GaAs-quantum-dot-InGaAsquantum-well structures is carried out where we observe an unusual dependence of the photoluminescence (PL) on the GaAs barrier thickness at strong magnetic field and excitation density. For the case of a thin barrier the quantum-well (QW) PL intensity is observed to increase at the expense of a decrease in the quantum-dot (QD) PL intensity. This is attributed to changes in the interplane carrier dynamics in the QW and the wetting layer (WL) resulting from increasing the magnetic field along with changes in the coupling between QD excited states and exciton states in the QW and the WL.
\end{abstract}

DOI: 10.1103/PhysRevB.100.035309

\section{INTRODUCTION}

Quantum-dot-quantum-well heterostructures represent a class of hybrid structures the photoluminescence (PL) wavelength of which depends on the dot sizes, well width, and dot-well barrier defining the strength of dot-well coupling. Variation of these parameters provides wide tunability in the engineering of these systems for optoelectronic applications including high-performance lasers, quantum information processors, or single-electron transistors [1-4]. In order to use them in high-speed tunnel injection quantum-dot (QD) lasers, the injected carriers are first collected by the quantum well $(\mathrm{QW})$, then tunnel into the QDs with subsequent relaxation to the ground state for laser action. By tunneling, cold carriers (electrons) from the QW transfer into the QD states without heating other carriers or phonons, thus reducing carrier leakage from the active region and, hence, increasing the differential gain in the lasers [5-7]. Optical properties of InAs/GaAs-InGaAs/GaAs dot-well structures have been extensively studied by means of steady-state and time resolved PL pump-probe measurements clarifying many issues of exciton dynamics [8-12].

Furthermore, application of a magnetic field to such structures allows for additional valuable information to be collected, because it introduces a strong but predictable change to the electronic structure $[13,14]$. At high magnetic fields, where the cyclotron energy is larger than both the lateral confinement energy and the exciton binding energy, the magnetic confinement dominates and a Landau-level-like structure is expected to develop. The magnetic field also removes the spin degeneracy giving rise to qualitatively different magnetic field dependences of the emission from the ground and excited

*Corresponding author: mdaldin@df.ufscar.br states, which is especially important in the case of dot-well structures, where excited QD states are brought into resonances with the QW ground or excited exciton states. Due to the strong QD-QW coupling in combination with spin properties, the spin injection dynamics of these hybrid structures has attracted great attention to the field of semiconductor spintronics [15-18]. In addition, its energy-level structure can be designed in order to improve the emission intensity at room temperature at telecommunication wavelengths [19]. Recently, many new results have been published for specific dot-well structures [20-26] outlining further perspectives on application of such hybrid structures.

Moreover, it was also revealed recently that the existence of indium-enriched islands in the InGaAs QW results in a spatially indirect (type-II) exciton. These excitons, electrons bound to a positively charged hole, inside a two-dimensional QW move in a ringlike orbit. As a result the PL intensity in InGaAs/GaAs QWs oscillates with magnetic field applied perpendicular to the QW plane at low temperatures. These oscillations have been attributed to the optical AharonovBohm effect associated with spatially indirect excitons that are formed in the vicinity of indium-rich InGaAs islands within the QW [27].

In this paper we have investigated the carrier coupling mechanisms at low temperature induced by a magnetic field in hybrid InAs/GaAs-QD-InGaAs-QW systems with different GaAs spacer layer thickness. The excitation density was intentionally increased to observe the third QD excited state, which is in resonance with the QW exciton ground state. The results from photoluminescence studies show that the hybrid structure with a thicker barrier behaves like the reference QD and QW structures, indicating that they are decoupled. On the other hand, the thinner barrier structure induces a strong coupling. We show that, at low magnetic fields, the coupling is preserved, whereas the enhancement of the QW in-plane 
confinement due to a higher applied magnetic field reduces the coupling and the luminescence behaves as two independent systems again.

\section{SAMPLE AND EXPERIMENTAL SETUP}

A set of hybrid InAs-QD- $\operatorname{In}_{0.13} \mathrm{Ga}_{0.87}$ As-QW samples was grown on semi-insulating GaAs (001) substrates in a Riber 32 molecular-beam epitaxy (MBE) system. Growth details are given in Ref. [10]. Each sample consists of a $0.3-\mu \mathrm{m}$ thick GaAs buffer layer, a 14-nm-thick InGaAs QW, a GaAs barrier with thickness $\left(d_{s p}\right)$ of 2 and $20 \mathrm{~nm}$, and a layer of self-assembled InAs QDs covered with a 50-nm GaAs cap layer. Two reference samples were grown under the same growth conditions as the dot-well structures. One is the self-assembled InAs QDs grown on the GaAs buffer layer and the other contains only a simple $\operatorname{In}_{0.13} \mathrm{Ga}_{0.87} \mathrm{As}$ QW. Structural analysis (not shown here) by transmission electron microscopy revealed the InAs QDs to be in the shape of platelets of approximately 5 -nm height and 20-nm diameter on average with an areal density of $10^{10} \mathrm{~cm}^{-2}$. The measured thickness of the QW was $14.0 \pm 0.5 \mathrm{~nm}$ in all samples and the GaAs spacer thickness was in good agreement with the parameters set during MBE growth.

Magnetophotoluminescence (MPL) measurements were performed at $4 \mathrm{~K}$ and with magnetic fields applied in Faraday geometry with magnitudes up to $9 \mathrm{~T}$ using a vibration-free helium closed cycle cryostat (Attocube/Attodry 1000) and a home made confocal microscope. A single mode optical fiber with $5-\mu \mathrm{m}$ core was used to bring a $660-\mathrm{nm}$ Toptica/Ibeam Series to a focus of $1-\mu \mathrm{m}$ spot and an excitation power of $90 \mu \mathrm{W}$. The luminescence from the sample was then collected by a multimode $50-\mu \mathrm{m}$ optical fiber before being dispersed by a $0.5-\mathrm{m}$ diffractive spectrometer and detected with an InGaAs diode array detector (Andor/Shamrock/Idus). Linear polarizers and half- or quarter-wave plates were properly set in order to identify the correspondent sigma plus $\left(\sigma^{+}\right)$and minus $\left(\sigma^{-}\right)$optical component emissions from all the samples.

\section{RESULTS AND DISCUSSION}

PL spectra measured from the reference QD sample at low temperature $(T=4 \mathrm{~K})$, high excitation power $(P=90 \mu \mathrm{W})$, and different magnetic fields $(B=0$ and $9 \mathrm{~T})$ are shown in Fig. 1(a). At low excitation power, $P=12.6 \mu \mathrm{W}$, the PL spectrum of the QDs (not shown) exhibits a single Gaussian emission with a maximum at $E=1.135 \mathrm{eV}$ and a full width at half maximum (FWHM) of $34 \mathrm{meV}$. At high power $(P=$ $90 \mu \mathrm{W})$ the reference QD spectrum transforms into the multiband spectral distribution shown in Fig. 1(a) with the blue line for zero magnetic field. Here the original ground state is found to redshift by $6 \mathrm{meV}$ due to many-body interaction in the QDs [28]. The additional bands which develop at the high-energy side of the ground-state Gaussian band are assigned to the dipole-allowed interband transition between excited QD states caused by state filling of the lower-energy levels in the QDs. As can be seen from Fig. 1(a) in the reference QD sample, a laser power of $90 \mu \mathrm{W}$ is enough to fill up to the second excited state at $1.261 \mathrm{eV}$. Excited-state bands are separated by $66 \mathrm{meV}$ and their FWHM ranges from 34 to $47 \mathrm{meV}$, as

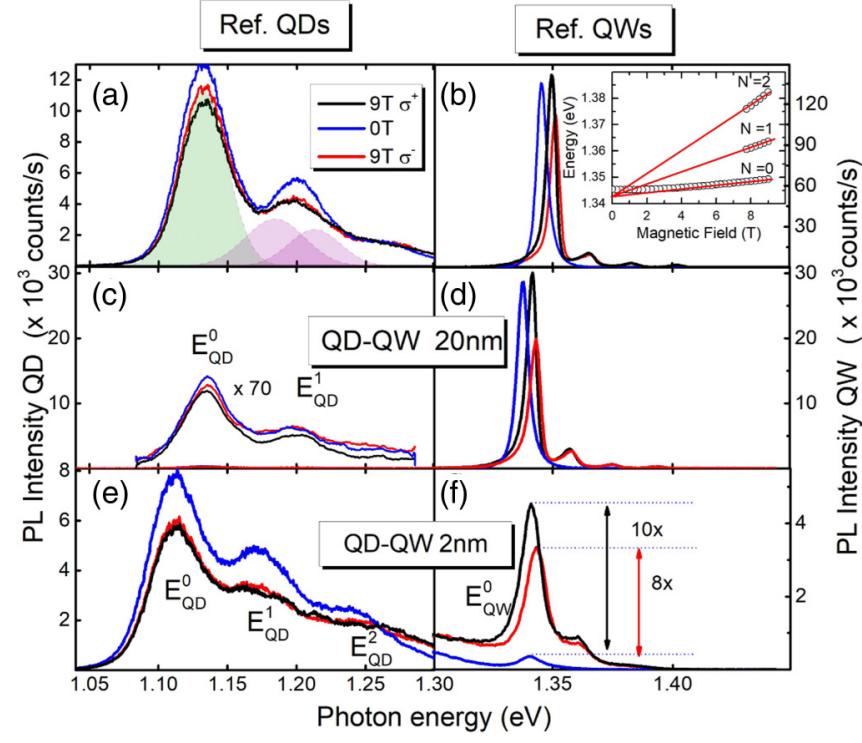

FIG. 1. PL spectra measured for (a) reference InAs QD and (b) reference $\mathrm{In}_{0.13} \mathrm{Ga}_{0.87} \mathrm{As} \mathrm{QW}$. The inset shows the LL splitting (solid lines are guides to the eye). Hybrid dot-well structures are shown with $d_{s p}=20 \mathrm{~nm}$ for (c) QD and (d) QW and with $d_{s p}=2 \mathrm{~nm}$ for (e) QD and (f) QW under high excitation power $P_{\mathrm{ex}}=90 \mu \mathrm{W}$. For all datasets are represented the detected polarizations $\sigma^{+}$(black lines) and $\sigma^{-}$(red lines) at $9 \mathrm{~T}$ and the spectra without magnetic field (blue lines). Gaussian fittings for the ground-state QD and first excited QD states are shown in Fig. 1(a).

deduced from a multiple Gaussian fit. This means that the first and second excited states are 66 and $132 \mathrm{meV}$ above the ground state, respectively. As the magnetic field is increased to $9 \mathrm{~T}$ the QD spectra exhibit a blueshift of the ground state of $1.2 \mathrm{meV}$, while the line shape and intensity of the excited states evolve as well. The asymmetry, broadening, and shift of the PL bands can be accurately fitted using multiple Gaussians. Such a fit is shown in Fig. 1(a) for $E_{\mathrm{QD}}^{0}$ and $E_{\mathrm{QD}}^{1}$ transitions in magnetic field $B=9 \mathrm{~T}$ for $\sigma^{-}$emission. In Ref. [13] it has been demonstrated that the first excited state can be split into two components related to break on the angular momentum degeneracy. Therefore, on this basis the first excited state was deconvoluted into two Gaussians and this splitting increases linearly with the magnetic field up to a value of $28.7 \mathrm{meV}$ at $B=9 \mathrm{~T}$. Comparing the spectra measured in \pm configurations, we measure the spin splitting of the QD states arising due to lifting of the spin degeneracy by the magnetic field. This splitting is given by $E=g \mu_{B} B$, where $g$ is the exciton $g$ factor and $\mu_{B}$ is the Bohr magneton.

The low-temperature $(T=4 \mathrm{~K}) \mathrm{PL}$ spectra measured for the reference $\operatorname{In}_{0.13} \mathrm{Ga}_{0.87} \mathrm{As} \mathrm{QW}$ under high excitation power $P=90 \mu \mathrm{W}$ for $B=0$ and $9 \mathrm{~T}$ are shown in Fig. 1(b). The low excitation PL spectrum (not shown here) has a maximum at $E=1.349 \mathrm{eV}$ and a FWHM value $2.95 \mathrm{meV}$ at zero field. This is the free exciton transition, $e_{1}-h h_{1}$, in the InGaAs QW. By increasing the magnetic field, the PL band blueshifts and some asymmetry is introduced. If the excitation intensity increases to $P=90 \mu \mathrm{W}$ the PL line shape becomes strongly dependent on magnetic field. High excitation power generates a high areal density of excitons in the QW resulting in Landau 
quantization at high field as shown in Fig. 1(b). Distinct peaks corresponding to transitions between Landau levels (LLs) allow us to draw fanlike plots of energies as a function of the magnetic field [inset Fig. 1(b)]. The total shift of the QW ground-state emission reaches $6 \mathrm{meV}$ at $9 \mathrm{~T}$, and the spin splitting between $\sigma^{+}$and $\sigma^{-}$polarizations is found to be $\sim 1.5 \mathrm{meV}$ at $B=9 \mathrm{~T}$.

Using our knowledge of the behavior of the reference samples in magnetic field we have investigated the hybrid dotwell structures where the QD and QW layers are separated by a GaAs barrier of different thicknesses. We expect here the PL properties of the constituent QD and QW layers to be similar to their reference examples, and will use this to understand the effects of the hybridization. Figure 1(c) shows the PL spectra of the dot-well sample with $d_{s p}=20 \mathrm{~nm}$ under magnetic field $(B=0$ and $9 \mathrm{~T})$ at the same high excitation power, under which the reference samples were measured [see Figs. 1(a) and 1(b)]. The 20-nm barrier is thick enough to assure weak direct dot-well coupling, such that the dot and well layers should be considered independent. Here we find the zero-field PL spectra to be very similar in position and shape to those of the reference samples. Noticeably, though, the integrated intensities are significantly lower for both the QD and QW layers. This implies that a thick GaAs barrier substantially attenuates the excitation on the hybrid structure. As a result we observe distinctly only the $E_{\mathrm{QD}}^{1}$ excited state of the QDs and a significantly weaker emission from the $e_{1}-h h_{1}$ in the QW. In addition, the magnetic field behavior becomes less pronounced for this 20-nm barrier sample. Nevertheless, the QD first excited level under $9 \mathrm{~T}$ is split in two components which can be separated by spectral deconvolution [Fig. 1(a)]. The PL spectra of the QW at 9 T show three LL emissions, which implies a high density of excitons in the QW [Fig. 1(d)]. As a result of these observations we conclude that for the thicker $\left(d_{s p}=20 \mathrm{~nm}\right)$ structure the QD and QW structures' responses are very much like the reference samples.

This changes dramatically for thin GaAs spacers $\left(d_{s p}=\right.$ $2 \mathrm{~nm}$ ). The hybrid structures with thinner barriers belong to the class of structures with strong direct coupling leading to a hybridization of the QD and QW excitonic states [8-12]. It has been shown and can be estimated from Fig. 1 that the spectral ranges corresponding to the QD third excited state and the QW ground state overlap each other $[10,12]$. As a result, due to the thin spacer the carriers can resonantly tunnel from the QW to the QD through the overlapped states, relax to the QD ground state, and emit in a spectral range that differs substantially from emission range of the QW. Figure 1(e) shows the PL spectra of the hybrid structure with $d_{s p}=2 \mathrm{~nm}$ measured with $P=90 \mu \mathrm{W}$ excitation without a magnetic field (blue lines) and at a magnetic field of $B=9 \mathrm{~T}$ in the $\sigma^{+}$and $\sigma^{-}$polarizations (black and red lines, respectively). In contrast to Fig. 1(c), the zero-field QD PL spectrum exhibits the pronounced structure of excited states up to $E_{\mathrm{QD}}^{3}$ indicating strong optical pumping to the QD layer leading to more PL intensity than seen in the hybrid structures $d_{s p}=20 \mathrm{~nm}$. At the same time, however, the zero-field QW emission is comparatively weaker and it vanishes completely at low power. This behavior indicates that the QW to QD carrier transfer is very efficient and completely depletes the population of the QW states at low excitation levels. Now, if the magnetic field

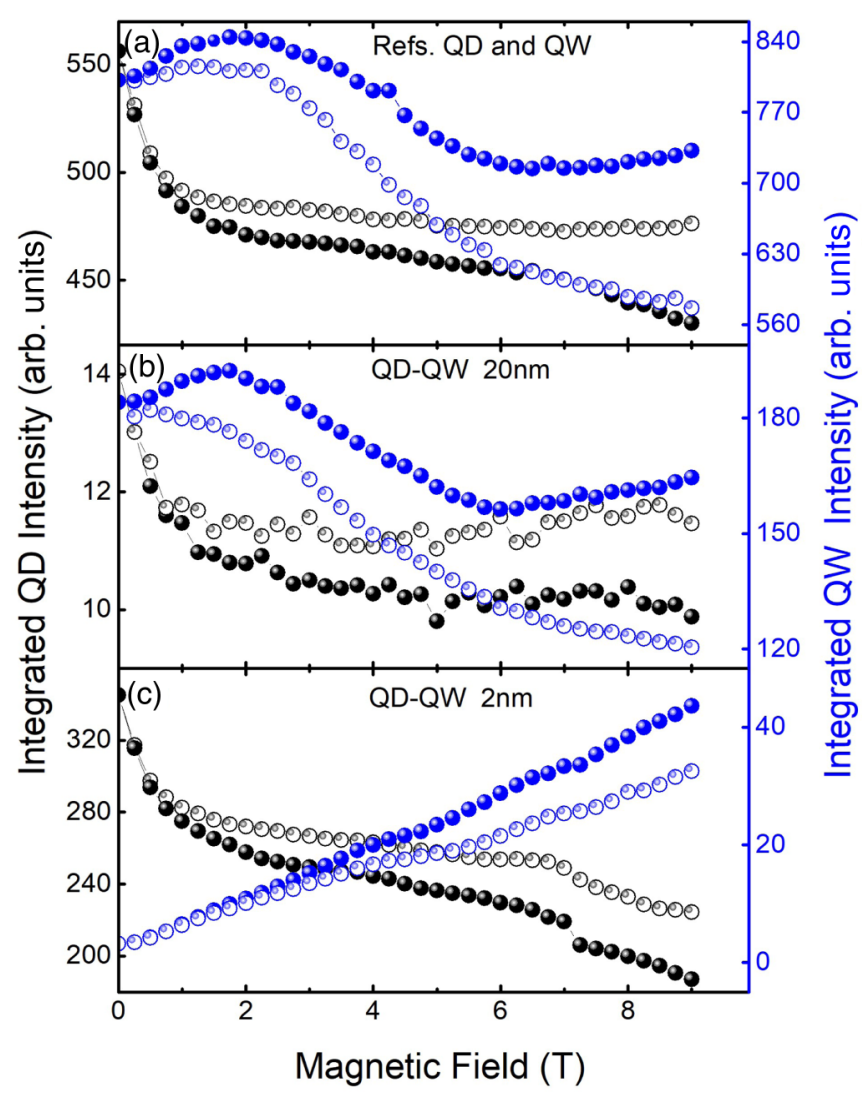

FIG. 2. (a) Integrated PL intensities for QD (left axes) and QW (right axes) ground state vs magnetic field measured in (a) reference QD and QW, (b) QD-QW hybrid dot-well structure separation $d_{s p}=$ $20 \mathrm{~nm}$, and (c) QD-QW hybrid dot-well structure with $d_{s p}=2 \mathrm{~nm}$ at $P_{\mathrm{ex}}=90 \mathrm{~W}$. Filled and hollow spheres represent the circular $\sigma^{+}$and $\sigma^{-}$polarized luminescence detection, respectively.

is applied at $B=9 \mathrm{~T}$, the QD emission gradually decreases, whereas the QW becomes high enough to develop even the $N=1$ LL transition in the QW PL spectrum [Fig. 1(f)].

Figure 2(a) shows that the integrated PL intensities for the QDs and QW reference samples as a function of the applied magnetic field become generally weaker at higher fields for both QD and QW nanostructures. For the QW a slight increase in the integrated intensity at low fields can be seen; however, as the magnetic field increases and the Landau levels appear, the FWHM (not shown here) starts to decrease and a drop in the integrated intensity is observed. Since the QD-QW system with $d_{s p}=20 \mathrm{~nm}$ [Fig. 2(b)] exhibits weak coupling, the same behavior of the integrated intensities versus magnetic field is observed.

The QDs and the QW integrated PL intensities as a function of the applied magnetic field for the hybrid structure with $d_{s p}=2 \mathrm{~nm}$ are shown in Fig. 2(c). By contrasting with the reference samples [Fig. 2(a)], the QD emission shows a comparable decrease in its intensity. On the other hand, for the QW emission, an intensity increase is observed, which is the opposite from what is depicted on the reference sample. We assume that such anticorrelated change of the integrated intensities indicates a very efficient magnetically controlled reduction of the dot-well coupling, which blocks the carrier 


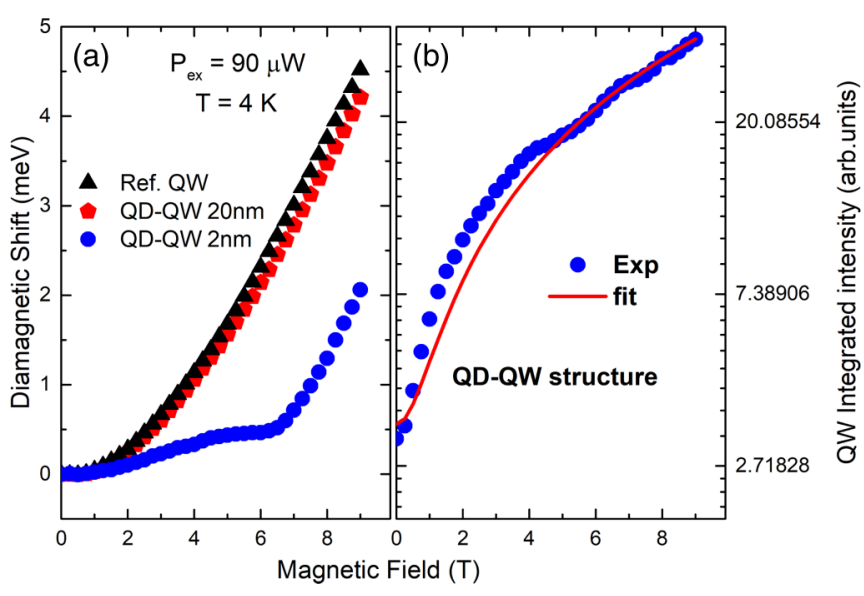

FIG. 3. (a) Diamagnetic shift vs magnetic field measured for the QW emission in the hybrid structures with $d_{s p}=2$ and $20 \mathrm{~nm}$ and in the reference QW at $P_{\text {ex }}=90 \mu \mathrm{W}$. (b) Comparison between WKB approximation and experimental QW integrated PL intensity.

transfer from the QW into the QD system, and thus enhances the QW PL by increasing the exciton density in the QW, while causing the QD PL to reduce by cutting off its exciton source.

The orbital Zeeman splitting of the QD states reaches only tenths of meV at a magnetic field of $9 \mathrm{~T}$ whereas the $\mathrm{QW}$ ground state shifts by $6 \mathrm{meV}$. We suggest that this difference in motion between the energy states of the two-dimensional (2D) QW and those of the confined QDs with magnetic field results in a strong detuning of the third QD excited state with the QW ground state. The tunneling then becomes nonresonant, which significantly lengthens the time of carrier transfer between the QW and QD systems and weakens the dot-well coupling. Figure 3(a) supports this conclusion. Here, the QW diamagnetic shift with magnetic field is shown for the reference QW and for the hybrid dot-well structures with different $d_{s p}$ values. It is known that strongly localized states exhibit smaller diamagnetic shifts than the less localized states [29]. The reference $\mathrm{QW}$ and the $\mathrm{QW}$ with $d_{s p}=20 \mathrm{~nm}$ both represent free 2D excitons with coinciding diamagnetic shifts. In the case of $d_{s p}=2 \mathrm{~nm}$, the diamagnetic shift reflects on the appearance of the dot-well coupling and reveals two ranges: below $6 \mathrm{~T}$, where the diamagnetic shift varies weakly indicating a regime of strong coupling and increased exciton localization, and above $6 \mathrm{~T}$, where the diamagnetic shift varies rapidly indicating a regime of decreasing localization which decouples the dot-well structures.

The observed decrease of the integrated PL intensity with magnetic field parallel to the growth direction has been observed before in arrays of InAs QDs in Ref. [30]. Here, it was established that the magnetic field in the Faraday geometry reduces the lateral transport to the dots since the field localizes carriers in wetting layer (WL) potential fluctuations with depths of a few meV. This formally means that the state population of the QDs at the same excitation power is lower with an applied magnetic field, while, at the same time, the population of the WL states increases. As a result, the QD emission is reduced, whereas the WL PL is enhanced [30]. We cannot completely exclude this mechanism in the quenching of our QD PL with magnetic field; however, at the best, it is not dominant in our case. Indeed, we do not observe any PL enhancement in the range of the WL transition $(1.45 \mathrm{eV})$. Moreover, the magnetic field can enhance the localization of carriers in the considerably deeper QD potential, as compared with WL potential fluctuations, thus favoring an increase of the QD PL intensity with increasing magnetic fields in Faraday geometry. Additionally the exciton lifetime reduces with an applied magnetic field, again contributing to the enhancement of the QD PL intensity.

Enhancement of a QW PL with a magnetic field has been observed as well [31] and attributed to the magnetic field induced compression of the wave function and corresponding increased oscillator strength. As a result the observed PL intensity was found to increase 1.5 times in an InGaAs/InP QW with an applied magnetic field of $7 \mathrm{~T}$. A compression of the in-plane wave functions of the carriers in a magnetic field perpendicular to the QW was also revealed in the time decay measurements of QWs [32]. This mechanism of QW PL enhancement cannot be ignored in our case, but it would not explain the eight- and tenfold [red and black arrows in Fig. 1(f)] increase of the PL amplitude observed in our hybrid structures.

There are several possibilities for the physical mechanisms of the magnetically reduced coupling observed in our hybrid dot-well structures. They must include jointly the effects of strain, confinement, and magnetic field on the valence band which contributes to the magnetoexciton state in the hybrid structure. This mechanism must also reproduce the experimentally observed ratio of the WL PL over the integrated PL intensity of the QDs which increases nonlinearly with the magnetic field, as well as the transport properties affecting the carrier capture by the QDs [33]. Of particular importance is the representation of the highly excited states of the InAs QDs in a magnetic field. Indeed it has been demonstrated that the overall pattern of the magnetic field evolution of the emission lines related to these states resembles a single-particle FockDarwin (FD) diagram [34].

Following Ref. [35] the excitonic energy shell structure of self-assembled InAs/GaAs quantum dots (taking into account Ga intermixing) can be found as a sum of electron and hole energies. For dipole-allowed transitions where only electrons and holes with the same set of quantum number recombine, a single-particle approximation to the excitonic FD spectrum is well reproduced as follows [35]:

$$
E\left(n_{+}, n_{-}\right)=E_{0}+\hbar \Omega_{+}(B)\left(n_{+}+\frac{1}{2}\right)+\hbar \Omega_{-}(B)\left(n_{-}+\frac{1}{2}\right)
$$

where $E_{0}$ consists of the vertical confinement energy and the semiconductor energy gap, and $\Omega_{ \pm}(B)=\Omega_{ \pm}^{e}(B)+\Omega_{ \pm}^{h}(B)$ is given by

$$
\Omega_{ \pm}(B)=\sqrt{\left(w^{2}+w_{c}^{2} / 4\right)} \pm\left|w_{c} / 2\right| .
$$

$n_{ \pm}=0,1,2,3, \ldots, w_{c}=e B / m^{*}$ is the cyclotron frequency and $w$ is the harmonic frequency, which describes the strength of the in-plane parabolic confinement. The splitting shifting and crossing may be qualitatively described by this excitonic FD spectrum for a thick GaAs barrier $\left(d_{s p}=20 \mathrm{~nm}\right)$ and the reference QD sample. Inclusion of many-body effects [35] lowers the energies of shells by $20 \mathrm{meV}$ and this lowering is larger for higher shells. The problem arises with 
the $n=3$ QD states that become strongly hybridized due to dot-well coupling for the thin barrier $\left(d_{s p}=2 \mathrm{~nm}\right)$. Taking into account several mechanisms which can contribute to the reduction of dot-well coupling in a magnetic field, let us consider our interpretation of carrier transfer in hybrid structures [9]. The results of measuring coherent tunneling between the InGaAs quantum well and InAs quantum dots by photoluminescence spectroscopy can be analyzed in terms of the Wentzel-Kramers-Brillouin (WKB) semiclassical approximation. The data were plotted as a function of the carrier transmission through the barrier as

$$
T(E) \propto \exp \left\{-\int_{0}^{d_{s p}}[V(x)-E]^{1 / 2} d x\right\}
$$

where $V(x)$ is the barrier offset potential and $E=E^{\text {exc }}$.

Recently, such an approximation has been widely used for the analysis of tunneling in generic asymmetric double-well potentials [36-38]. In our case the QW state shifts quadratically under the magnetic field, $B$. Therefore we will fit our transmission using the form

$$
T(B) \propto \exp \left(\sqrt{\alpha+\beta B^{2}}\right) .
$$

Figure 3(b) shows the integrated PL intensity of the QW in the hybrid InAs/InGaAs structure versus the magnetic field $B$ in a semilogarithmic plot. The fit using Eq. (4) is plotted also. Good agreement between experiment and the WKB approximation gives evidence that the diamagnetic shift of the QW exciton is a dominant contribution to a reduction of the dot-well coupling in our hybrid structures.

\section{CONCLUSIONS}

In this paper we carried out a MPL study of the carrier transfer in hybrid InAs/GaAs-quantum-dot-InGaAsquantum-well structures with varying barrier thickness. The measurements were performed with the magnetic field parallel to the growth direction (the Faraday geometry) up to $9 \mathrm{~T}$ and low temperature $(4 \mathrm{~K})$. At excitation densities sufficiently high to observe the QW PL we found a strong dependence of the QW PL intensity on magnetic field both for weak and strong coupling between the dots and the well. The observed exchange of PL intensity from the QDs to the QW with magnetic field is attributed to the breaking of their resonant coupling. This is the result of a change of in-plane carrier dynamics in both the QW and WL. Both the formation of Landau levels and the change of coupling between QD excited states and exciton states in the QW and the WL due to their different diamagnetic shifts ultimately bring them out of resonance.

\section{ACKNOWLEDGMENTS}

The authors gratefully acknowledge the financial support of the following agencies: NSF Grants No. OIA1457888; CSIC Grant No. I-COOP-2017-COOPB20320; MINEICO Grant No. EUIN2017-88844; FAPESP Grants No. 2013/18719-1, No. 2014/07375-2, No. 2014/19142-2, and No. 2018/01914-0; CNPq Grant No. 164765/2018-2 and CAPES (Finance Code 001).
[1] D. L. Huffaker, G. Park, Z. Zou, O. B. Shchekin, and D. G. Deppe, Appl. Phys. Lett. 73, 2564 (1998)

[2] D. Bimberg, Semiconductor Nanostructures (Springer, New York, 2008).

[3] Semiconductor Spintronics and Quantum Computation, edited by D. D. Awschalom, D. Loss, and N. Samarth (Springer, Berlin, 2002).

[4] K. K. Likharev, Proc. IEEE 87, 606 (1999).

[5] G. Walter, N. Holonyak, J. H. Ryou, and R. D. Dupuis, Appl. Phys. Lett. 79, 3215 (2001)

[6] P. Bhattacharya, S. Ghosh, S. Pradhan, J. Singh, Z.-K. Wu, J. Urayama, K. Kim, and T. B. Norris, IEEE J. Quantum Electron. 39, 952 (2003).

[7] Z. Mi, P. Bhattacharya, and S. Fathpour, Appl. Phys. Lett. 86, 153109 (2005).

[8] Y. I. Mazur, B. L. Liang, Z. M. Wang, D. Guzun, G. J. Salamo, Z. Y. Zhuchenko, and G. G. Tarasov, Appl. Phys. Lett. 89, 151914 (2006).

[9] Y. I. Mazur, V. G. Dorogan, D. Guzun, E. Marega, Jr., G. J. Salamo, G. G. Tarasov, A. O. Govorov, P. Vasa, and C. Lienau, Phys. Rev. B 82, 155413 (2010).

[10] Y. I. Mazur, V. G. Dorogan, M. E. Ware, E. Marega, Jr., M. Benamara, Z. Y. Zhuchenko, G. G. Tarasov, C. Lienau, and G. J. Salamo, Nanoscale 4, 7509 (2012).

[11] Y. I. Mazur, V. G. Dorogan, E. Marega, Jr., D. Guzun, M. E. Ware, Z. Y. Zhuchenko, G. G. Tarasov, C.
Lienau, and G. J. Salamo, J. Appl. Phys. 113, 034309 (2013).

[12] D. Guzun, Y. I. Mazur, Jr., V. G. Dorogan, M. E. Ware, E. Marega,Jr., G. G. Tarasov, C. Lienau, and G. J. Salamo, J. Appl. Phys. 113, 154304 (2013).

[13] P. P. Paskov, P. O. Holtz, B. Monemar, J. M. Garcia, W. V. Schoenfeld, and P. M. Petroff, Phys. Rev. B 62, 7344 (2000).

[14] R. A. Child, R. J. Nicholas, and N. J. Mason, Phys. Status Solidi B 238, 281 (2003).

[15] X.-J. Yang, T. Kiba, T. Yamamura, J. Takayama, A. Subagyo, K. Sueoka, and A. Murayama, Appl. Phys. Lett. 104, 012406 (2014).

[16] Y. Q. Huang, I. A. Buyanova, X. J. Yang, A. Murayama, and W. M. Chen, Phys. Rev. Appl. 9, 044037 (2018).

[17] K. Takeishi, S. Hiura, J. Takayama, K. Itabashi, M. Urabe, A. Washida, T. Kiba, and A. Murayama, Phys. Rev. Appl. 10, 034015 (2018).

[18] H. Chen, S. Hiura, J. Takayama, S. Park, K. Sueoka, and A. Murayama, Appl. Phys. Lett. 114, 133101 (2019).

[19] W. Rudno-Rudziński, M. Syperek, J. Andrzejewski, E. Rogowicz, G. Eisenstein, S. Bauer, V. I. Sichkovskyi, J. P. Reithmaier, and G. Sęk, Sci. Rep. 8, 12317 (2018).

[20] S. Bauer, V. Sichkovskyi, F. Schnabel, A. Sengül, and J. P. Reithmaier, J. Cryst. Growth 516, 34 (2019).

[21] S. L. Chen, T. Kiba, X. J. Yang, J. Takayama, and A. Murayama, J. Appl. Phys. 119, 115701 (2016). 
[22] S. L. Chen, T. Kiba, X. J. Yang, J. Takayama, and A. Murayama, Appl. Phys. Lett. 108, 152103 (2016).

[23] K. Połczyńska, E. Janik, P. Kossacki, and W. Pacuski, Acta Phys. Pol. A 132, 369 (2017).

[24] M. Pieczarka, M. Syperek, D. Biegańska, C. Gilfert, E. M. Pavelescu, J. P. Reithmaier, J. Misiewicz, and G. Sęk, Appl. Phys. Lett. 110, 221104 (2017).

[25] M. Syperek, J. Andrzejewski, E. Rogowicz, J. Misiewicz, S. Bauer, V. I. Sichkovskyi, J. P. Reithmaier, and G. Sęk, Appl. Phys. Lett. 112, 221901 (2018).

[26] I. Khanonkin, G. Eisenstein, M. Lorke, S. Michael, F. Jahnke, A. K. Mishra, and J. P. Reithmaier, Phys. Rev. B 98, 125307 (2018).

[27] L. Schweidenback, T. Ali, A. H. Russ, J. R. Murphy, A. N. Cartwright, A. Petrou, C. H. Li, M. K. Yakes, G. Kioseoglou, B. T. Jonker, and A. Govorov, Phys. Rev. B 85, 245310 (2012).

[28] R. Heitz, F. Guffarth, I. Mukhametzhanov, M. Grundmann, A. Madhukar, and D. Bimberg, Phys. Rev. B 62, 16881 (2000).

[29] C. Schulhauser, D. Haft, R. J. Warburton, K. Karrai, A. O. Govorov, A. V. Kalameitsev, A. Chaplik, W. Schoenfeld,
J. M. Garcia, and P. M. Petroff, Phys. Rev. B 66, 193303 (2002).

[30] M. Larsson, E. S. Moskalenko, L. A. Larsson, P. O. Holtz, C. Verdozzi, C.-O. Almbladh, W. V. Schoenfeld, and P. M. Petroff, Phys. Rev. B 74, 245312 (2006).

[31] M. Sugawara, N. Okazaki, T. Fujii, and S. Yamazaki, Phys. Rev. B 48, 8848 (1993).

[32] I. Aksenov, Y. Aoyagi, J. Kusano, T. Sugano, T. Yasuda, and Y. Segawa, Phys. Rev. B 52, 17430 (1995).

[33] S. Ménard, J. Beerens, D. Morris, V. Aimez, J. Beauvais, and S. Fafard, J. Vac. Sci. Technol. B 20, 1501 (2002).

[34] A. Babinski, M. Potemski, S. Raymond, J. Lapointe, and Z. R. Wasilewski, Phys. Rev. B 74, 155301 (2006).

[35] S. Raymond, S. Studenikin, A. Sachrajda, Z. Wasilewski, S. J. Cheng, W. Sheng, P. Hawrylak, A. Babinski, M. Potemski, G. Ortner, and M. Bayer, Phys. Rev. Lett. 92, 187402 (2004).

[36] G. Rastelli, Phys. Rev. A 86, 012106 (2012).

[37] V. Benderskii, E. Vetoshkin, and H. Trommsdorff, Chem. Phys. 244, 299 (1999).

[38] A. Garg, Am. J. Phys. 68, 430 (2000). 